\title{
Real-Time Simulation of 4D Lung Tumor Radiotherapy Using a Breathing Model
}

\author{
Anand P. Santhanam ${ }^{1,2}$, Twyla Willoughby ${ }^{2}$, Amish Shah ${ }^{2}$, Sanford Meeks ${ }^{2}$, \\ Jannick P. Rolland ${ }^{1}$, and Patrick Kupelian ${ }^{2}$ \\ ${ }^{1}$ College of Optics and Photonics, University of Central Florida \\ jannick@odalab.ucf.edu \\ ${ }^{2}$ Department of Radiation Physics, M.D. Anderson Cancer Center Orlando \\ \{Anand.santhanam, twyla.willoughby, amish.shah, \\ Sanford.meeks, Patrick.kupelian \}@orhs.org
}

\begin{abstract}
In this paper, we present a real-time simulation and visualization framework that models a deformable surface lung model with tumor, simulates the tumor motion and predicts the amount of radiation doses that would be deposited in the moving lung tumor during the actual delivery of radiation. The model takes as input a subject-specific 4D Computed Tomography (4D CT) of lungs and computes a deformable lung surface model by estimating the deformation properties of the surface model using an inverse dynamics approach. Once computed, the deformable model is used to simulate and visualize lung tumor motion that would occur during radiation therapy accounting for variations in the breathing pattern. A radiation treatment plan for the lung tumor is developed using one of the 4D CT phases. During the simulation of radiation delivery, the dose on the lung tumor is computed for each beam independently.
\end{abstract}

\section{Introduction}

Physics and physiology based lung deformation models have been extensively discussed in application areas ranging from computer aided diagnostics to medical image processing. Recent advances in 4D medical imaging modalities have led to effective medical applications by coupling medical simulations with patient-specific 4D anatomical models and their physically and physiologically realistic lung deformations. Of particular importance is the application of these deformable models for areas related to radiotherapy. Lung tumors move during breathing in unpredictable trajectories that depend on patient's body orientation and physiological condition. The uncertainty in tumor location compromises the accurate deposition of radiation doses during radiotherapy. This is particularly a concern when high radiation doses are delivered with the intent of ablating tumors and subsequently gaining local regional control of tumor growth in an environment that includes radiation sensitive structures such as normal lung tissue and the esophagus.

In this paper, we present a method to model, simulate and visualize the radiation dose delivery on a moving $3 \mathrm{D}$ lung tumor. The contributions of this paper are (a) a 
novel method to model the 3D lung tumor motion, and (b) the integration of the lung tumor motion with a treatment plan simulator. Specifically, the lung surface motion is modeled using a physics-based and physiology-based approach. This deformable lung model allows us to account for variations in breathing conditions. A novel method to model the lung tumor motion based on the lung surface's deformation is discussed in this paper. A dose accumulation model based on pre-calculated doses is used to calculate the delivered dose to various aspects of the moving tumor during radiotherapy. Results show the effect of subject-specific tumor motion on its dose accumulation and the observed computational time. These real-time simulations and visualizations of the 3D lung tumor dose accumulation would enable the real-time monitoring of radiation delivery and the use of high focused radiation fields that could result in decreased treatment related toxicity.

There have been many studies to evaluate the motion of a lung tumor for radiation therapy. 4D CT facilitates the availability of the patient specific lung tumor motion details. A detailed discussion on the usage of 4D CT for predicting tumor motion is given in the report of the AAPM Task-Group 76 (TG 76) [3]. 4D CT scans or multiple CT scans at different phases of the respiratory cycle have been used to assess the range of target motion and to more adequately define the margins for motion.[4] Once the target motion has been described, a dose model was used to combine the probability distribution of the target motion with the treatment machine's pencil beam fluence to calculate dose $[1,5,6]$. Additionally, 3D optical flow analysis of 4D CT datasets and direct dose accumulation method was used to compute the lung tumor motion and the dose accumulated on the lung tumor, respectively [2]. These calculations, although accurate for the CT scan on which they are calculated, may not be applicable during therapy as the lung displacement captured from the 4D CT may differ from the lung displacement during the radiation therapy. These differences are caused by variations in breathing such as rib-cage diaphragm compensation and changes in lung ventilation during radiation delivery. A physics and physiology based lung model, which is extracted from 4D CT can simulate the lung deformation and the lung tumor motion during the treatment. A key requirement to obtain subject-specific lung deformation is to estimate the elastic properties that control the deformation. Inverse deformation (ID) methods allow the estimation of elastic tissue parameters of a 3D object with its known 3D deformation and applied force [10]. The general approach for the ID methods is to associate a mathematical representation such as Green's function (GF) to the 3D model's shape change and estimate the deformation parameters.

\section{Materials and Methods}

In this section, we discuss a simulation framework that takes into account the subjectspecific lung surface and tumor motion extracted from 4D CT images (Siemens Biograph 64) using a physics and physiology based approach and the radiation plan (iPlan, Brainlab) prescribed for the patient using a direct dose accumulation method.

\subsection{Estimation of a Deformable Lung Surface Model}

We now briefly describe the inverse dynamic step taken in the estimation of subjectspecific deformation operator. We use the deformation method discussed in [8], 
which has been validated using 4D CT and also has been applied for the PET-CT registration [7], for the inverse analysis. The method used for estimating the deformation operator for the lung was first described in [9] and is now summarized for the comprehensiveness of the paper. The general formula of the GF as an operator in discrete space dot product is given as

$$
x(I)=\sum_{J}^{N}\left[\frac{C_{I}}{4 \pi\left(A_{I} d(J, I)+B_{I} e(J, I)\right)}\right] \times f(J),
$$

where $x(I)$ is the magnitude of the displacement of the node $I, f(J)$ is the magnitude of the force applied on node $J, N$ is the number of nodes (approximately 150,000 nodes), $C_{I}$ is an arbitrary constant that which depends on the deformation mechanics of the lungs. $A_{I}$ and $B_{I}$ are referred to as structural constants as they depend on the 3D lung model's structure. The function $d(J, I)$ represents the Euclidean distance between the nodes $J$ and $I$. The function $e(J, I)$, which represents the elastic interaction between nodes $J$ and $I$, is given as a difference in the alveolar expansion of the region surrounding nodes $J$ and $I$. This function is based on the physiological fact that the air flows to the region of least resistance, which in our case is the region of higher alveolar expandability. The regional alveolar expandability is thus an indirect indicator of the Young's Modulus (YM). The regional alveolar expandability has been previously discussed in [8]. A method to estimate the values of $A_{I}, B_{I}$, and $C_{I}$ using a forward lung dynamics approach [8] is discussed in [9].

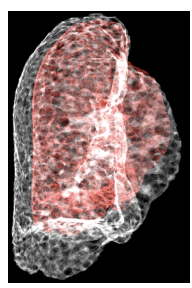

(a)

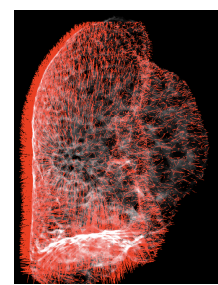

(b)

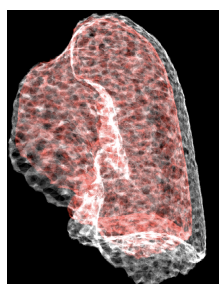

(c)

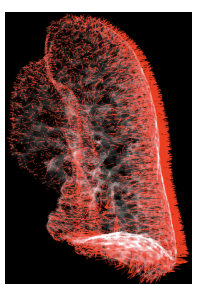

(d)

Fig. 1. Lung deformation is shown for lungs as an overlap of the lung at the start of inhalation (red model) and the deformed lung at the end of inhalation (grey model)

The steps involved in the inverse deformation analysis are as follows: A 4D CT data set taken at the intervals of $10 \%$ of inspiration volume was used as input and the 3D lungs were segmented from each 3D CT of this dataset using Osirix software. The lung tumors are segmented by clinical experts. The CT noise was removed using the Laplacian smoothing algorithm. A set of 5 3D CT datasets was chosen for the estimation of the GF operator and the rest of the 3D CT datasets (5) are used for validation. The selected 3D segmented 3D lung surface models chosen were then registered to each other using the method discussed in [8]. Once registered, the displacement vector for every lung surface node are known. The applied force on each node was taken to be the vertical gradient of the lung orientation. The values of $A_{I}, B_{I}$, and $C_{I}$ were computed as discussed in [9]. Fig. 1a and c shows the initial (red model) and deformed (grey model) lungs. Fig. $1 \mathrm{~b}$ and $1 \mathrm{~d}$ shows the direction vectors of the left and right lung surface 
nodes The deformations are separately validated by comparing the simulated shapes for a given air volume with 3D CT snapshots at the same air volume.

\subsection{Modeling Lung Tumor Motion}

We now describe a novel method to compute subject-specific lung tumor motion accounting for the subject-specific lung deformation operator estimated in $\S 2.1$. This method is based on the fact that the lung tumor acts as a single rigid body connected to the lung tissue. Since our deformable lung model consists only of the lung surface, an association is made between the point in the center of the tumor and the lung surface nodes. We create a data structure that stores a surface node location and the angle of this surface node made from the center of the lung tumor center. The data structure is populated by projecting a ray from the center of the tumor and computing a ray triangle intersection with the lung surface model. From a physics perspective, each of these rays represent springs such that the tumor is connected to the lung surface by the springs. The closest node of intersection point is noted. Let $p(\theta, \vartheta)$ be a 3D lung surface node in the polar coordinate $(\theta, \vartheta)$. Let $d(p(\theta, \vartheta))$ be the normalized Euclidean distance between the center of the lung tumor and the surface node $p(\theta, \vartheta)$. The normalization factor in this case is the Euclidean distance between the center of the lung tumor and the farthest lung surface node. It can be written as

$$
d(p(\theta, \vartheta))=1-\frac{d_{e}(p(\theta, \vartheta))}{d_{e}^{\max }}
$$

where $d_{e}$ is the Euclidean distance between the center of the lung tumor and the surface node and $d_{e}{ }^{\max }$ is the Euclidean distance between the center of the lung tumor and the farthest lung surface node. Let $l$ be an interpolation function, which can be written as

$$
l(\theta, \vartheta)=\frac{d(p(\theta, \vartheta))}{d(p(\theta, \vartheta))+d(p(\pi+\theta, \vartheta))} \cdot
$$

It can be seen that the interpolation function is based on the Euclidean distance of the tumor center from two lung surface points given by $p(\theta, \vartheta)$ and $p(\pi+\theta, \vartheta)$. The displacement vector $x_{T}$ of the lung tumor motion is computed from the displacement vector of the lung surface nodes $X(\theta, \vartheta)$ as

$$
{ }^{X} T=\frac{1}{N} \sum_{\theta=0 \vartheta=0}^{\pi} \sum^{2 \pi} l^{2}(\theta, \vartheta) X(\theta, \vartheta) .
$$

From equation (4), it can be seen that the tumor is affected more by the surface nodes that are closer to it than the nodes that are far from it.

\subsection{Treatment Plan and Delivery}

We now discuss a simulation framework that integrates the 3D deformable lung model (§2.1) and the lung tumor motion (\$2.2). We first discuss the steps involved in developing a treatment plan using a single 3DCT scan. These plans generated using the 
Brainlab treatment planning system used five or seven coplanar radiation beams to deliver a dose of $40 \mathrm{~Gy}$ to the edge of the target as defined using a single CT scan with no margin. A 3D dose matrix for each individual radiation beam in the region around the target volume was extracted for use in the model. The purpose of individually extracting each beam was to simulate the method in which each beam was delivered in the patient and to match the radiation beam geometry to the patient motion.

We now discuss the integration of the 3D deformable lung model (\$2.1) and the lung tumor motion (\$2.2) with the 3D dose grids extracted from the treatment plan developed for the patient. The simulation framework consists of a virtual cuboid of dimension 100X100X100 cubes created with each cube of the dimension 1X1X1 $\mathrm{mm}^{3}$. A 3D sphere representing the lung tumor is then introduced in the virtual cuboid. The center of this 3D sphere is located at the lung tumor position. The sphere center is modeled to move based on the lung surface movement as described in \$2.2. The deformable lung surface model discussed in $\$ 2.1$ is generally controlled using spirometry measurements of the pressure-volume curve. In this simulation framework, we use a strain gauge belt (Anzai Medical Co.) to provide the subject-specific breathing signal to the deformable model. We simultaneously measure the rib-cage movements using the strain gauge and slow vital capacity using a spirometer for correlating the change in lung volume and the strain gauge signal. The simulation framework computes the real-time beam delivery by calculating the time to deliver each beam of radiation from machine characteristics so that the motion of the target during each beam can be simulated. The doses from each beam are summed to the moving target one beam at a time the same way in which radiation treatment is delivered. At each discrete time step, the overlap between the voxels of the tumor and the dose matrix cube is computed. The overlap of the dose matrix with the moving tumor is computed using a level-set based binary searching algorithm for the first time and neighborhood searching method as the tumor moves. Once the overlapping dose matrix cube is computed, the dose in the tumor voxel is added with the dose from that dose matrix cube divided by the total number of time steps.

\section{Simulation Results}

In this section, we first show the impact of the generic tumor motion on dose accumulation. We then show the impact of subject-specific tumor motion on the dose accumulation. Finally, we discuss the computational time taken by the simulator. The simulation framework that includes the estimation of deformable lung surface model (\$2.1), the estimation of lung tumor motion (\$2.2) and the simulation of dose delivery (§2.3) were implemented using an Intel Core 2 Duo $2.16 \mathrm{GHz}$ laptop. The source code for the computation and visualization was developed using $\mathrm{C}++\&$ OpenGL. Fig. 2a represents the dose that was deposited to the 3D sphere without the introduction of motion. The 3D dose accumulation illustrates the regions of therapeutic dose ( $>95 \%$ of central dose: White), underdose ( $85-90 \%$ of central dose: Green), and partial treatment (90-95\% of central dose: Blue). The black zone inside the 3D sphere represents the region with less than $85 \%$ of the central dose. This therapeutic dose region was intentionally made tight around the target for illustration. It can be seen 


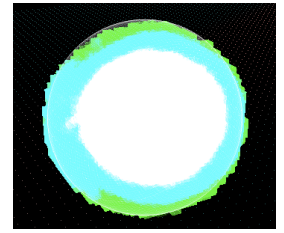

(a)

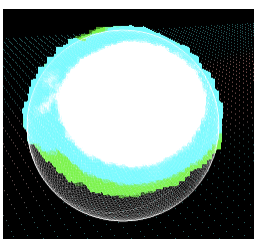

(b)

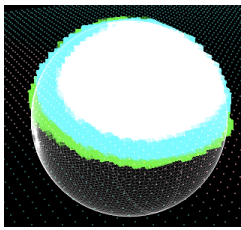

(c)

Fig. 2. "Dose accumulation in tumor with (a) no motion, (b) $1 \mathrm{~cm}$ amplitude of motion and (c) $2 \mathrm{~cm}$ amplitude of motion."

that the high dose region is large for the 3D tumor with no motion. Fig. 2b and Fig. 2c represents the dose distributions that were accumulated to the sphere as the target was moved at $1 \mathrm{~cm}$ and $2 \mathrm{~cm}$ amplitude, respectively. The green area represents the clinically underdosed area (85-90\% radiation), the blue area represents doses between $90 \%$ and $95 \%$ of the maximum. It can be seen that the amount of dose inside the tumor decreases with increase in motion.

Results shown in Fig. 3 show the visualization of the lung surface model with the lung tumor motion simulated based on the surface model's motion. In this case, the strain gauge connected to the patient is used as a driver for the surface lung model deformation. Fig. 3a shows 2D CT snapshot of the left lung with tumor in the lower lung and Fig $3 \mathrm{~b}$ represent the lung surface contour and lung tumor contour. The effect of the lung tumor motion on the dose delivery can be seen in Fig. 3c and 3d. Fig. 3d uses a 3D spherical mesh of the same radius as that of the tumor to illustrate the dose accumulation. The tumor motion was observed to be more along the superior-inferior direction than the anterior-posterior direction. Since the radiation beams were along the anterior-posterior direction, the top region of the lung tumor in this case has received higher dose, while the rest of the lung tumor has received reduced amounts of dose. Fig. 3e shows a 2D CT snapshot of the right lung with tumor in the middle lung and Fig $3 \mathrm{f}$ represents the lung surface contour and lung tumor contour. The effect of the lung tumor motion on the dose delivery can be seen in Fig. 3g and 3h. The tumor motion in this case was observed to be more along the anterior-posterior direction as compared to the superior-inferior direction, which resulted in higher radiation dose accumulation than the previous case. It can be seen that the amount of dose delivered on the lung tumor varies with the location of the tumor because tumor motion varies with position in the lungs. The difference in tumor motion and size causes the lung tumor in the upper lung (Fig. 3d) to receive fewer doses than the lung tumor in the lower lung (Fig. 3h). The real-time nature of the lung surface deformation as well as the tumor motion enables the real-time simulation of lung tumor dosimetry. The lung deformation is performed using GPU at 60 frames-per-second (FPS). Together with the simulation of the lung tumor motion and the tumor dose accumulation, the integrated simulation framework performs at 40 FPS satisfying real-time requirements for even advanced visualization methods. The FPS increased to 70 as the number of lung surface model nodes was reduced to 75,000. Additionally, it can be seen that the dose accumulation is done using more breathing phase snapshots (200 frames per aver age breathing) than 4D CT, which provides higher accuracy in dose accumulation estimations. 




(a)

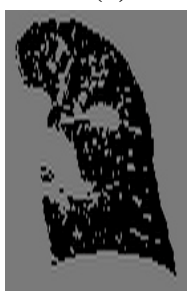

(e)

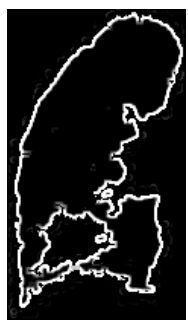

(b)

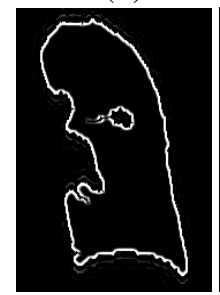

(f)

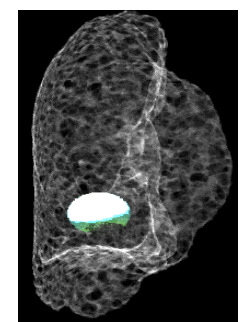

(c)

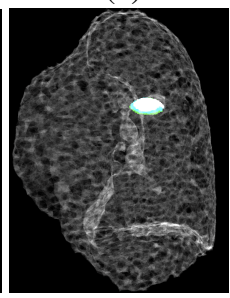

(g)

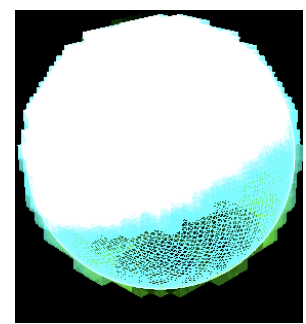

(d)

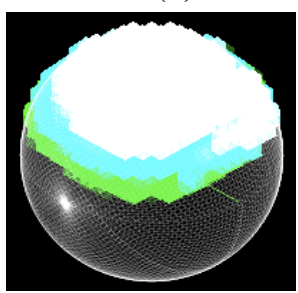

(h)

Fig. 3. Lung tumor on the lower left lung (a-d) and upper right lung (e-h). The CT images of the lungs with the tumor are shown in (a) and (e). The lung surface contours are shown in (b) and (f). The tumor dose delivery is shown in (c) and (g). The lung tumor displacement is simulated as shown in 2.2. The tumor dose accumulation with the spherical boundary is shown in (d) and (h).

\section{Discussion}

We describe a method for simulating and visualizing the lung surface deformation, tumor motion and its dosimetry. The proposed method compensates for the limitations in the phantom based dosimetry studies as it can allow computation of 3D dosimetry and analysis. The proposed method also compensates for the limitations in the 4D CT based analysis because it can take into account tumor movement at an increased number of frames per breath and the breathing variations during treatment. Future work will involve a detailed discussion on all the registration and segmentation methods and their effect on lung deformation modeling. Future work will employ Monte Carlo algorithms to accurately account for dose differences associated with the normal lung and the lung tumor region.

\section{References}

1. Chetty, I.J., Rosu, M., Neelam, T., Marsh, L.H., McShan, D.L., Balter, J.M., Fraass, B.A., Ten Haken, R.K.: A fluence convolution method to account for respiratory motion in three-dimensional dose calculations of the liver: a Monte Carlo study. Medical Physics 7, 1776-1780 (2003)

2. Guerrero, T., Zhang, G., Segars, W., Huang, T., Bilton, S., Ibott, G., Dong, L., Forster, K., Ping Lin, K.: Elastic image mapping for 4-D dose estimation in thoracic radiotherapy. Radiation Protection Dosimetry 115(1), 497-502 (2005) 
3. Keall, P.J., Mageras, G.S., Balter, J.M., Emery, R.S., Forster, K.M., Jiang, S.B., Kapatoes, J.M., Low, D.A., Murphy, M.J., Murray, B.R., Ramsey, C.R., Van Herk, M.B., Vedam, S.S., Wong, J.W., Yorke, E.: The management of respiratory motion in radiation oncology report of AAPM Task Group 76. Medical Physics 33(10), 3874-3900 (2006)

4. Low, D.A.: A method for the re-construction of four dimensional synchronized CT scans acquired during free breathing. Medical Physics 30(6), 1254-1263 (2003)

5. Low, D.A., Parikh, P.J., Lu, W., Dempsey, J.F., Wahab, S.H., Hubenschmidt, J.P., Nystrom, M.M., Handoko, M., Bradley, J.D.: Novel breathing motion model for radiotherapy. International Journal of Radiation Oncology Biology Physics 63(3), 1776-1780 (2005)

6. Lu, W.: Real-time respiration monitoring using the radiotherapy treatment beam and four dimensional computed tomography (4DCT)- a conceptual study. Physics in Medicine and Biology 51(18), 4469-4495 (2006)

7. Moreno, A., Chambon, S., Santhanam, A., Brocardo, R., Kupelian, P., Rolland, J.P., Angelini, E., Bloch, I.: Thoracic CT-PET registration using a 3D breathing model. Medical Image Computing and Computer Aided Intervention, 626-633 (2007)

8. Santhanam, A., Fidopiastis, C., Imielinska, C., Rolland, J.P.: Modeling and simulation of Real-time 3D lung dynamics. IEEE Transactions on Information Technology and Biomedicine 12(2), 257-270 (2008)

9. Santhanam, A.P., Mudur, S.P., Rolland, J.P.: An inverse 3D lung deformation analysis for medical visualization. In: Computer Animation and Social Agents, Geneva, Switzerland, pp. 229-242. Computer Graphics Society (2006)

10. Wilson, L.S., Robinson, D.E., Dadd, M.J.: Elastography-the movement begins. Physics for Medicine and Biology 45, 1409-1421 (2000) 